\title{
Expression of the pluripotency marker UTF1 is restricted to a subpopulation of early $A$ spermatogonia in rat testis
}

\author{
Maaike P A van Bragt ${ }^{1}$, Hermien L Roepers-Gajadien ${ }^{1}$, Cindy M Korver ${ }^{2}$, Jan Bogerd ${ }^{1}$, \\ Akihiko Okuda ${ }^{3}$, Bart J L Eggen ${ }^{4}$, Dirk G de Rooij ${ }^{1,2}$ and Ans M M van Pelt ${ }^{2}$ \\ ${ }^{1}$ Department of Endocrinology and Metabolism, Faculty of Science, Utrecht University, 3584 CH Utrecht, \\ The Netherlands, ${ }^{2}$ Center for Reproductive Medicine, Academic Medical Center, 1105 AZ Amsterdam, \\ The Netherlands, ${ }^{3}$ Division of Developmental Biology, Research Center for Genomic Medicine, \\ Saitama Medical University, 1397-1 Yamane, Hidaka, Saitama 350-1241, Japan and \\ ${ }^{4}$ Department of Developmental Genetics, Groningen Biomolecular Sciences and Biotechnology Institute, \\ University of Groningen, 9751 NN Haren, The Netherlands
}

Correspondence should be addressed to A M M van Pelt at Center for Reproductive Medicine, Academic Medical Center, Room F2-131-2, Meibergdreef 9, 1105 AZ Amsterdam, The Netherlands; Email: a.m.vanpelt@amc.uva.nl

\begin{abstract}
The population of early A spermatogonia includes stem cells that possess spermatogonial stem cell properties. Recent reports suggest that these cells have the ability to regain pluripotent properties. Here, we show that expression of the pluripotency marker undifferentiated embryonic cell transcription factor 1 (UTF1) is restricted to distinct germ cells within the testis. In embryonic and neonatal testes, all gonocytes were found to strongly express UTF1. During further testicular development, expression of UTF1 was restricted to a subset of A spermatogonia and with the increase in age the number of cells expressing UTF1 decreased even more. Ultimately, in the adult rat testis, only a small subset of the A spermatogonia expressed UTF1. Remarkably, even in testes of vitamin A-deficient rats, in which the early $A$ spermatogonia $\left(A_{s}, A_{p r}\right.$ and $\left.A_{a l}\right)$ are the only type of spermatogonia, only a subset of the spermatogonia expressed UTF1. In the adult rat testis, expression of UTF1 is restricted to a subpopulation of the ZBTB16 (PLZF)-positive early A spermatogonia. Furthermore, the observed distribution pattern of UTF1expressing cells over the different stages of the cycle of the seminiferous epithelium suggests that the expression of UTF1 is restricted to those $A_{s}, A_{p r}$ and short chains of $A_{a l}$ spermatogonia that are in the undifferentiated state and therefore maintain the ability to differentiate into $A 1$ spermatogonia in the next round of the epithelial cycle or possibly even in other directions when they are taken out of their testicular niche. Reproduction (2008) 136 33-40
\end{abstract}

\section{Introduction}

Spermatogenesis is a continuous process starting with spermatogonial stem cells (de Rooij \& Russell 2000, Aponte etal. 2005), which were until recently thought of as unipotent stem cells only being able to form cells of the spermatogenic lineage (Hofmann etal. 2005, Smith 2006). However, recent reports suggest that these cells have a greater potential. Testes of both neonatal and 6-week-old mice were reported to contain pluripotent cells or cells able to obtain these properties in vitro (Kanatsu-Shinohara et al. 2004, Guan et al. 2006, Seandel et al. 2007).

Previously, it was described that primordial germ cells (PGCs), of both human and mouse origin, could give rise to pluripotent stem cells (Matsui et al. 1992, Shamblott et al. 1998). Finally, PGCs, gonocytes, and spermatogonial stem cells express several genes believed to be essential for pluripotency, e.g., Pou5f1 (previously octamer-binding transcription factor 3/4, Oct3/4) and Sry box 2 (Sox2) (Pesce et al. 1998, Shi et al. 2006).
Another pluripotency-associated gene is Utf1 (Okuda et al. 1998). This gene was found to be expressed in embryonic stem (ES) cells, embryonic carcinoma (EC) cells, and PGCs (Okuda et al. 1998, Nishimoto et al. 1999, Chuva de Sousa Lopes et al. 2005). In adult mice, Utf1 mRNA was only detected in the ovary and testis (Okuda et al. 1998). In EC and ES cells, protein expression of UTF1 was found to be localized to the nucleus and subnuclear fractionation, and mobility assays revealed that UTF1 was a chromatin-associated protein with histone-like properties (van den Boom et al. 2007). Differentiation of EC cells is accompanied by a rapid reduction in UTF1 levels, indicating a stem cell-specific function (Okuda et al. 1998, Nishimoto et al. 1999, van den Boom et al. 2007). Furthermore, it was suggested that activation of UTF1 is an important mechanism by which POU5F1 maintains the stem cell state of ES cells (Nishimoto et al. 2005). Experiments indicated that UTF1 plays a role in the proliferation rate and the teratoma-forming capacity of ES cells (Nishimoto et al. 2005). However, recently it was 
proposed that UTF1 might be involved in the maintenance of a specific epigenetic profile that allows lineage-specific differentiation of ES and EC cells while it is not involved in stem cell renewal (van den Boom et al. 2007).

In order to learn more about a possible role of UTF1 in spermatogenesis, we now have studied the localization of UTF1 in the testis at different developmental stages during pre- and postnatal life. During testicular development, the expression of UTF1 was restricted to the gonocytes and A spermatogonia. Cell counts revealed a decrease in the number of UTF1-positive cells during testicular development and expression in the adult rat testis was found to be restricted to a subpopulation of early A spermatogonia.

\section{Results}

\section{Utf1 mRNA is expressed in testis of neonatal, pubertal, and adult rats}

To determine Utf1 mRNA expression in the rat testis of different ages, we first obtained the rat Utf1 sequence. Adult rat total testis RNA was subjected to RT-PCR using primers derived from the human and mouse Utf1 cDNA sequences.
The resulting rat $U t f 1 \mathrm{cDNA}$ sequence (GenBank acc. no. EU176857) encodes a predicted protein of 338 amino acids and comparison between rat and mouse or human UTF1 showed an overall amino acid identity of 93 and $65 \%$ and similarity of 96 and $73 \%$ respectively (Fig. 1). Previously, based on the human and mouse UTF1 protein sequences, two conserved domains, CD1 and CD2, were identified (Fukushima et al. 1998). Analysis of the corresponding domains in rat UTF1 revealed identities of 96 and $87 \%$ for CD1 and 100 and $86 \%$ for CD2 compared with mouse and human UTF1 respectively (Fig. 1).

To determine Utf1 expression during rat testicular development, RT-PCRs were performed on total testis RNA of rats of various ages (Fig. 2). Utf1 mRNA was detected in neonatal, pubertal, and adult rat testes and in the testes of vitamin A-deficient (VAD) rats (Fig. 2).

\section{Gonocytes and spermatogonia express UTF1}

To determine which cell types in the testis express UTF1, we performed immunohistochemistry at different developmental stages during pre- and postnatal life. At 16 days post coitum $(\mathrm{dpc})$, a strong staining for UTF1 was present in the nuclei of all gonocytes (Fig. 3A).

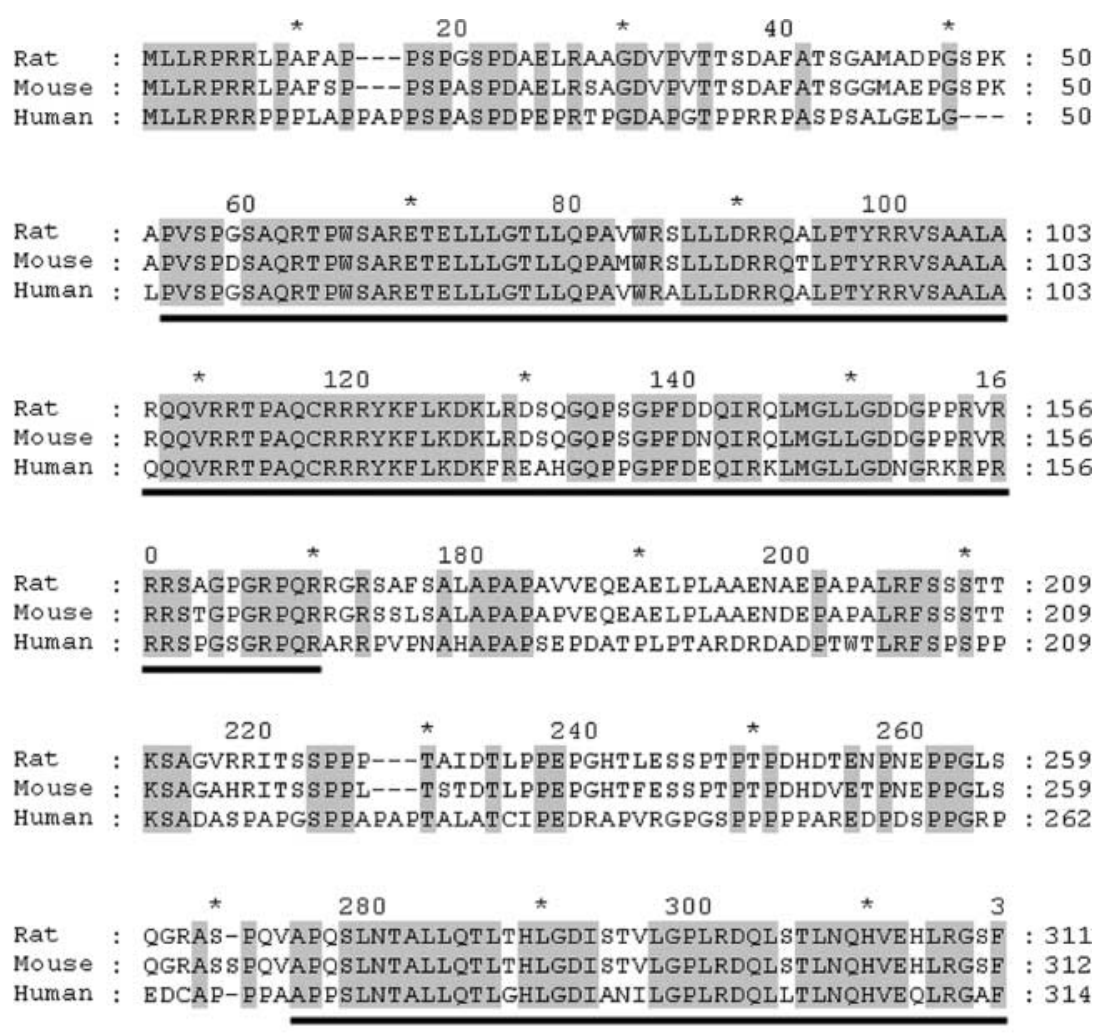

Rat : $\begin{array}{ll}20 & * \quad 340 \\ & \text { DQTVSLAVGEILGSAASERGILGDLRQ }\end{array}$

Mouse: DQTVSLAVGEILGSAASERGILGDLRQ : 339

Human : DQTVSLAVGEILGSAAAERGVLRDPCQ : 341
Figure 1 Amino acid sequence of rat UTF1 protein compared with the mouse and the human UTF1 protein sequences. Identities are marked by the gray boxes. The two conserved domains are underlined (Fukushima et al. 1998). 


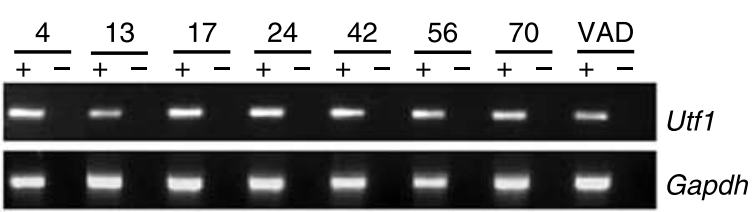

Figure 2 RT-PCR for Utf1 on total testis of rat of several ages and VAD total testis. Numbers express age in days post partum. +, cDNA; - , RT control.

In gonocytes undergoing mitosis, the condensed chromosomes were stained for UTF1 (Fig. 3A). At 18 $\mathrm{dpc}$ and 4 days post partum (dpp), the expression was also strong and localized to the nuclei of all gonocytes (Fig. 3B and C). At 9 dpp, when gonocytes have migrated to the basal membrane and have become spermatogonia, UTF1 expression was detected in the nuclei of almost all A spermatogonia (Fig. 3D). At 13 dpp, staining was present in A spermatogonia, but not in B spermatogonia or preleptotene spermatocytes (Fig. 3E). At later stages during development and in adult rat testes, when spermatogenesis was complete, UTF1 expression was restricted to A spermatogonia (Fig. 3F-J). However, in testes of older rats, A spermatogonia that did not express detectable UTF1 levels were also observed. In VAD animals, seminiferous tubules only contain early $A$ spermatogonia $\left(A_{s}, A_{p r r}\right.$ and $\left.A_{a l}\right)$, Sertoli cells and sporadically preleptotene spermatocytes (Mitranond et al. 1979, Van Pelt \& De Rooij 1990). Immunohistochemistry showed that in the VAD rat testis, UTF1 is expressed in spermatogonia (Fig. 3K). Interestingly, also in VAD testes, spermatogonia were observed that did not show expression of UTF1. Immunolocalization experiments were repeated with three other antibodies directed against UTF1, all confirming the localization of UTF1 to the nuclei of gonocytes and A spermatogonia. In accordance with the immunolocalization of UTF1 to the spermatogonia in VAD rat testes, RT-PCR results confirmed the expression of Utf1 in spermatogonia isolated from these testes (Fig. 4). We furthermore found that the early A spermatogonia marker Zbtb16 (previously promyelocytic leukemia zinc finger, PLZF) and the activators of Utf1 transcription Pou5f1 and Sox2 were also expressed by the early A spermatogonia isolated from the VAD rat testes (Fig. 4).

\section{The number of UTF1-positive cells decreases with age}

The expression pattern of UTF1 during testicular development indicated that the number of UTF1-positive cells in the testis decreased with age. To quantify this finding, the

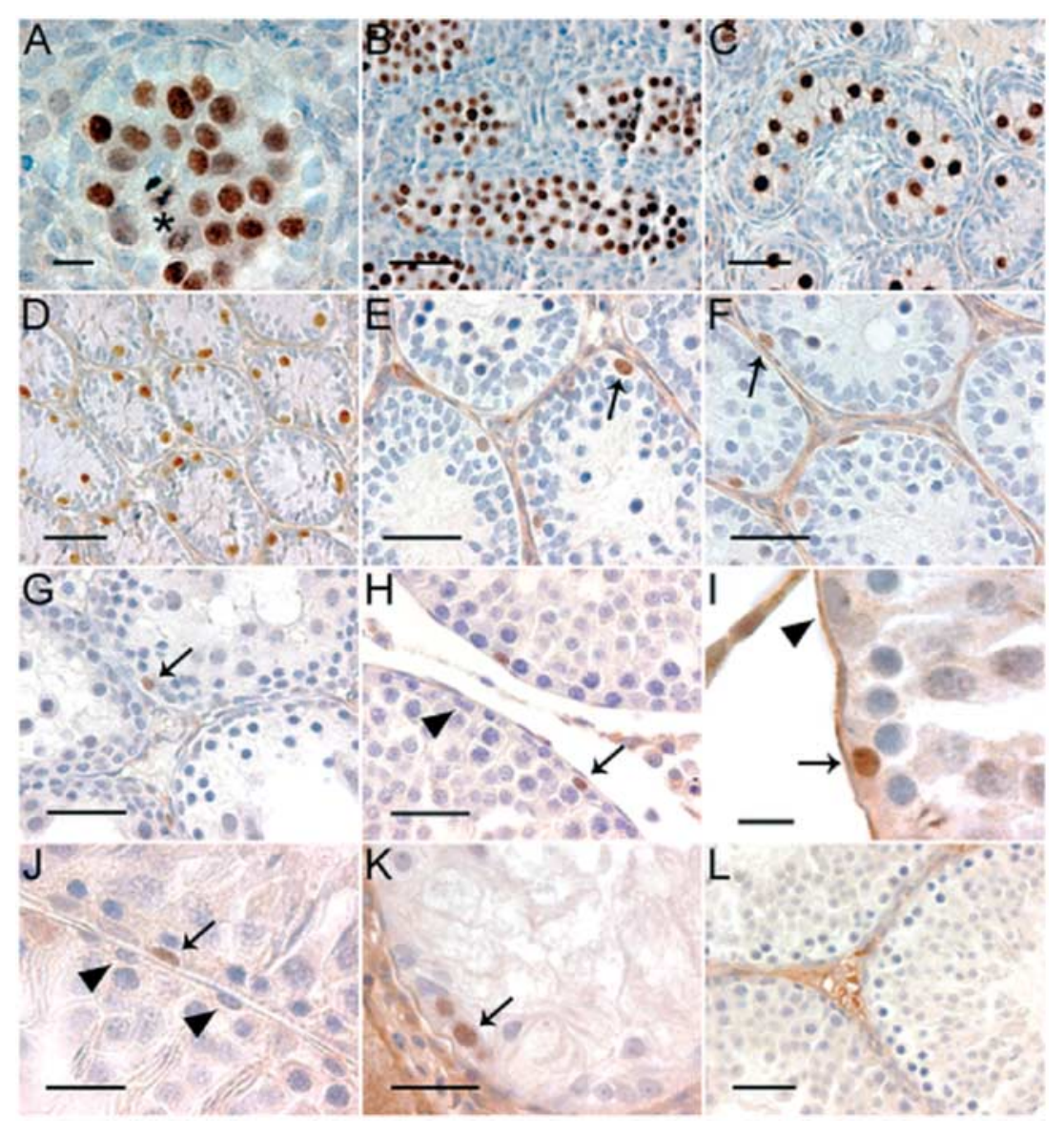

Figure 3 UTF1 expression in rat testes of different ages. (A) $16 \mathrm{dpc}$, (B) $18 \mathrm{dpc}$, (C) $4 \mathrm{dpp}$, (D) $9 \mathrm{dpp}$, (E) $13 \mathrm{dpp}$, (F) $17 \mathrm{dpp},(\mathrm{G}) 24 \mathrm{dpp}$, (H) $42 \mathrm{dpp}$, (I) $56 \mathrm{dpp}$, (J) $70 \mathrm{dpp}$, (K) adult VAD rat, and (L) negative control $70 \mathrm{dpp}$. Asterisk, mitotic gonocytes; arrows, positive spermatogonia; arrowheads, negative spermatogonia. Bars: (A and I) $100 \mu \mathrm{m},(B-D$ and L) $40 \mu \mathrm{m},(\mathrm{E}-\mathrm{H}, \mathrm{J}$ and K) $50 \mu \mathrm{m}$. Figure shows the immunolocalization of UTF1 performed with the AB3383 antibody. 


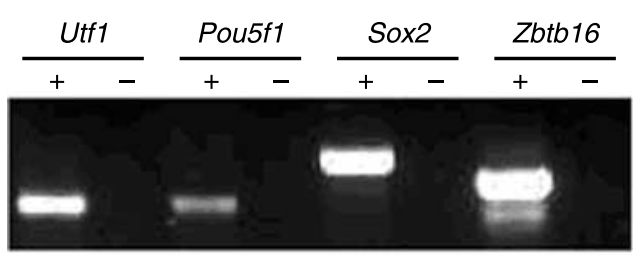

Figure 4 RT-PCR for Utf1, Pou5f1, Sox2, and Zbtb16 (PLZF) on spermatogonia isolated from VAD rat testis. +, cDNA; -, RT control.

numbers of cells expressing UTF1 and ZBTB16 were determined. ZBTB16 is a transcriptional repressor known to be required for the self-renewal of spermatogonial stem cells and its expression is known to be restricted to early $\mathrm{A}$ spermatogonia (Buaas etal. 2004, Costoya etal. 2004). The numbers of UTF1- and ZBTB16-positive cells per tubule cross-section were scored in adjacent sections of rat testis of different ages (Fig. 5). While the numbers of ZBTB16positive cells increased during testicular development, the number of UTF1-positive cells clearly decreased. Indicating that in the adult rat, there is a clear reduction in UTF1-positive A spermatogonia, whereas the number of ZBTB16-positive A spermatogonia increased during testicular development.

\section{UTF1 expression is restricted to a subpopulation of early $A$ spermatogonia}

To study which type of A spermatogonia expressed UTF1, we performed double immunofluorescent labeling for UTF1 and ZBTB16. At 42 dpp, almost all ZBTB16-positive spermatogonia also expressed UTF1. However, at 56 and $70 \mathrm{dpp}$, not all ZBTB16-positive spermatogonia also expressed UTF1 (Fig. 6). Cells positive for UTF1 but negative for ZBTB16 were not observed. These findings indicate that in the adult rat testis, UTF1 expression was restricted to a subpopulation of early A spermatogonia.

\section{Pattern of the UTF1-positive cells during the epithelial cycle}

Determining which early A spermatogonia express UTF1 is extremely difficult as early A spermatogonia are morphologically indistinguishable (de Rooij 1998). However, the numbers of the different types of early A spermatogonia follow a specific pattern during the

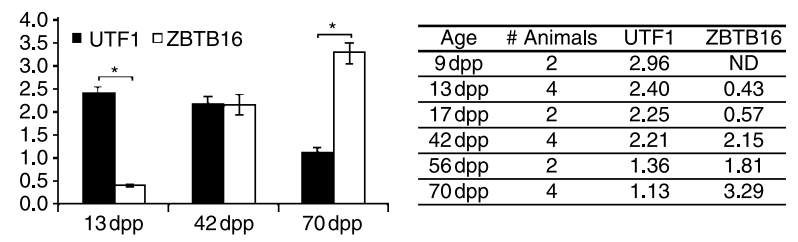

Figure 5 Number \pm S.E.M. of UTF1- and ZBTB16-positive cells per tubule cross-section in the testes of rats of various ages. ND, not determined. Asterisk indicates that a significant difference $(P \leq 0.05)$ between the number of UTF1- and ZBTB16-positive cells. epithelial stages. Studying the numbers of UTF1expressing cells during the epithelial stage could thus possibly reveal the identity of UTF1-expressing cells.

The numbers of UTF1-positive spermatogonia per tubule cross-section were scored for the different epithelial stages (Fig. 7). During epithelial stages VII-X, the highest number of UTF1-positive spermatogonia was observed. The number decreased slowly from stages X until II-III, after which an increase in the numbers of UTF1-positive cells was found during stages IV-VI. However, the numbers in stages IV-VI did not yet reach the level observed in stage VII. In comparison, the average number of ZBTB16positive spermatogonia per tubule cross-section remained relatively constant with exception of the decrease observed in stage XIV (Fig. 7). During all stages, the number of ZBTB16-positive spermatogonia was higher than the number of spermatogonia that expressed UTF1. At stage VII, the biggest overlap between UTF1 and ZBTB16 expression was observed.

\section{Discussion}

The mechanisms whereby spermatogonial stem cells self-renew, differentiate, and possibly maintain their pluripotent potential are not yet fully known. In order to unravel the underlying mechanisms, the identification of proteins involved in one or more of these processes is crucial. The pluripotency-associated protein UTF1 is a prime candidate for being involved in the process of selfrenewal and/or differentiation of spermatogonial stem cells and possibly in maintaining the pluripotent potential of these cells.

The rat sequence of the Utf1 gene was found to be highly identical to that of mouse, but considerably different from the human sequence (Fukushima et al. 1998). The identities of the conserved domains are higher compared with the overall identity and therefore the rat sequence supports the existence of the two conserved domains (Fukushima et al. 1998). Utf1 mRNA expression was found in the testes of both developing as well as the adult rats, indicating that Utf1 expression is not restricted to a certain developmental stage.

In embryonic and postnatal (4 dpp) rat testis, expression of UTF1 protein was found to be restricted to the gonocytes. Following further testicular development, expression of UTF1 was only observed in A spermatogonia. However, the number of cells per tubule cross-section that expressed UTF1 decreased with age, and non-expressing type A spermatogonia were observed in the adult rat testes, suggesting that not all types of A spermatogonia expressed UTF1.

Even in the testes of VAD rats, in which the early A spermatogonia are the only type of spermatogonia, expression of UTF1 was restricted to the spermatogonia. This finding was supported by RT-PCR which showed expression of Utf1 and its upstream factors Pou5f1 and Sox2 in A spermatogonia isolated from VAD rat testes. 


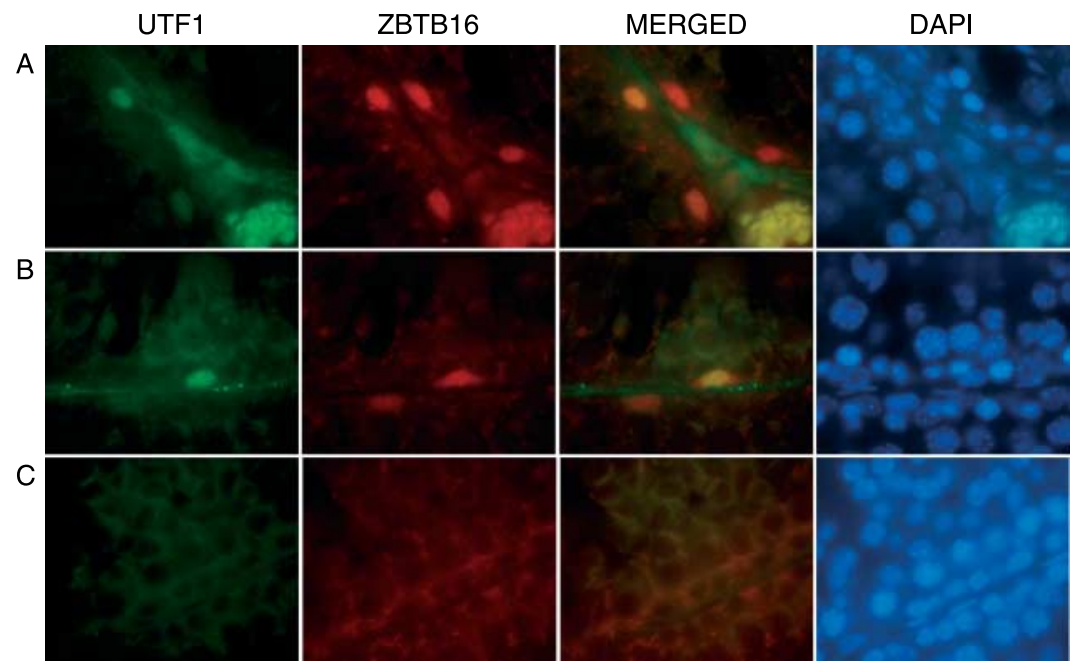

Figure 6 Co-localization of UTF1 and ZBTB16 in 56 dpp rat testes. (A and B) Partial co-localization of UTF1 and ZBTB16 can be observed in the testis of two different 56 dpp rats. (C) Negative control.
Surprisingly, however, in VAD rat testis spermatogonia that did not express UTF1 were also observed, indicating that UTF1 expression was restricted to a subpopulation of the early A spermatogonia.

As the different types of A spermatogonia are morphologically very difficult to distinguish, co-localization studies of UTF1 with ZBTB16 were performed in order to reveal the identity of the UTF1-positive cells. In mice, expression of ZBTB16 in the testis is known to be restricted to early A spermatogonia (Buaas et al. 2004, Costoya et al. 2004). To our knowledge, localization of ZBTB16 in rat testes has not been described before. The expression pattern of ZBTB16 we observed in both crosssections as well as in whole-mount seminiferous tubules of rat testes was similar to the expression seen in mice (data not shown). In addition, we determined the number of ZBTB16-positive spermatogonia during the cycle of the seminiferous epithelium, which to our knowledge has not been shown before.

The co-localization studies of UTF1 with ZBTB16 showed an almost complete overlap in A spermatogonia of $42 \mathrm{dpp}$ old rats. However, in adult (56 and 70 days old) rats only partial co-localization was observed; cells that expressed UTF1 always expressed ZBTB16, but cells expressing ZBTB16 did not always express UTF1. These observations are in accordance with our cell counts of the numbers of UTF1- and ZBTB16-positive cells per tubule cross-section in the testes of rats of various ages (Fig. 5). At $42 \mathrm{dpp}$, when in almost all cells UTF1 and ZBTB16 are co-expressed, counting revealed equal numbers of UTF1and ZBTB16-expressing cells. At 10 weeks, approximately three times more ZBTB16-expressing cells were counted compared with the number of cells expressing UTF1, thereby confirming the co-localization experiments that not all ZBTB16-positive cells expressed UTF1.

Thus, expression of UTF1 in the adult rat testis is restricted to a subpopulation of early A spermatogonia as shown by the lower number of UTF1-positive cells compared with the number of ZBTB16-positive cells, the partial co-localization of UTF1 with ZBTB16, and the observation that in the testis of VAD rats not all early $A$ spermatogonia expressed UTF1.

The important question then is which types of early A spermatogonia in the adult rat testis expressed UTF1. Unfortunately, our attempts to immunohistochemically stain for UTF1 on whole mounts of seminiferous tubules were not successful, not allowing us to identify the UTF1-expressing spermatogonia. In order to shed more light on this issue, we determined the number of UTF1-positive spermatogonia during the cycle of the seminiferous epithelium. A pattern was found in which the number of UTF1-positive spermatogonia peaked in stages VII through $\mathrm{X}$, gradually decreasing thereafter until stage III, and then increasing again. This pattern is quite different from that of the total numbers of early $\mathrm{A}$ spermatogonia throughout the epithelial cycle that shows an increase from about stages $X$ to III, almost similar numbers from stages III until VII and sharply decreasing thereafter as the $A_{a l}$ spermatogonia differentiate into A1 spermatogonia (Huckins 1971). As $A_{a l}$ spermatogonia form the largest group within the population of early A spermatogonia, their pattern is

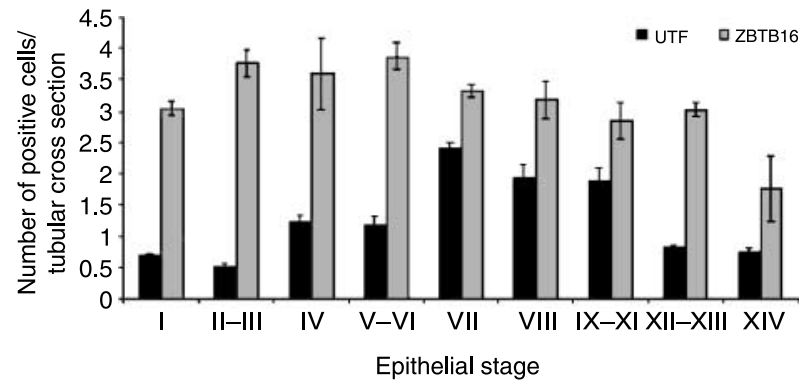

Figure 7 Average number \pm s.E.M. of UTF1- and ZBTB16-positive cells per tubule cross-section for the different epithelial stages in 10-week-old rat testes. 
similar to this latter pattern and thus does not reflect the pattern of the UTF1-expressing cell numbers we observed in this study. Surprisingly, the observed expression pattern of UTF1 also does not reflect the pattern of the numbers of $A_{s}$ or/and $A_{p r}$ spermatogonia that remains about the same during the epithelial cycle (Huckins 1971). Furthermore, very few divisions of early A spermatogonia occur from epithelial stages IV until IX (de Rooij 1998). Thus, as hardly any new early A spermatogonia are formed, the increase in UTF1-positive spermatogonia observed in epithelial stage VII, cannot be explained by the generation of new UTF1-positive cells by mitotic activity of existing UTF1-positive cells. It rather suggests that the observed pattern is related to the function of UTF1 and that its expression is stage specifically induced or upregulated to detectable levels in existing early A spermatogonia during epithelial stages IV-VII.

Recently, it was reported that in EC and ES cells UTF1 has histone-like properties and it was postulated to be involved in maintaining stem cells in a specific epigenetic profile, either by attracting chromatin-modifying proteins or by chromatin compaction (van den Boom et al. 2007). In the testis, UTF1 could have a similar function as in EC and ES cells. It is therefore most likely that the increase in the number of UTF1-positive cells during stages II-III until VII represent the $A_{s}, A_{p r r}$ and $A_{a l}$ spermatogonia that do not differentiate into $A 1$ spermatogonia but rather go through another round of the epithelial cycle. The observed distribution of UTF1positive cells over the different epithelial stages supports this idea, as the number of UTF1-positive spermatogonia in stages II-III reflects the number of $A_{s}$ and $A_{p r}$ spermatogonia as determined by Huckins for this stage (Huckins 1971). Furthermore, the early A spermatogonia that during stage VII did not differentiate, do not undergo mitotic divisions until stage $X$ (de Rooij 1998). The decrease in the number of UTF1-positive cells per tubule cross-section thus coincides with the mitotic divisions of the early A spermatogonia in stage $\mathrm{X} / \mathrm{XI}$, suggesting that UTF1 expression is gradually lost from the population of early A spermatogonia as new or larger syncytia of $A_{a l}$ spermatogonia are generated.

Therefore, we propose that those $A_{s}, A_{p r}$ and short chains of $A_{a l}$ spermatogonia that do not differentiate into A1 spermatogonia express UTF1 and that these spermatogonia thus maintain the ability to differentiate in the next round of the epithelial cycle into A1 spermatogonia or possibly even in other directions when they are taken out of their testicular niche.

Recent reports described the presence of pluripotent stem cells in both the neonatal and the adult mouse testis (Kanatsu-Shinohara et al. 2004, Guan et al. 2006). A point of debate was whether these stem cells possessed pluripotency capacity in vivo, or whether these cells acquired their pluripotency in vitro. However, noncultured cells isolated from adult mouse testes were shown to be able to contribute to multiple tissues upon injection into blastocysts, indicating the presence of pluripotent stem cells in the adult mouse testis (Guan et al. 2006). Since UTF1 is thought to play a role in maintaining the pluripotent capacity and our immunohistochemistry experiments localize the expression of UTF1 to the population of cells that are close to, if not identical to the population of germ cells with stem cell properties, our results suggest that cells with the capacity for pluripotency are present in the testis during all stages of development, including the adult stage. However, our results furthermore suggest that with increasing age the number of stem cells with the capacity for pluripotency decreases and that the isolation and culture of sufficient numbers of these cells will be more difficult from the testes of adult animals than that of young animals, an observation supported by findings previously reported by Kanatsu-Shinohara et al. (2004).

In conclusion, we have shown that during all stages of testicular development expression of UTF1 is restricted to the population of germ cells that contain the stem cells. The distribution of UTF1-positive cells over the different epithelial stages suggests that UTF1 in the testis plays a role in maintaining the cells in an undifferentiated state that allows differentiation into one or more directions. We therefore believe that further studies on the function of UTF1 in the testis will reveal more insight into the mechanism(s) of spermatogonial stem cell selfrenewal and the potential pluripotent capacity.

\section{Materials and Methods}

\section{Animals}

Testes from Wistar rats of different ages (16 and $18 \mathrm{dpc}, 4,9,13$, $17,24,42,56$, and $70 \mathrm{dpp}$ ) were snap frozen in liquid nitrogen or fixed for $6 \mathrm{~h}$ in $4 \%$ formaldehyde, followed by overnight fixation in diluted Bouin's fluid (71\% (v/v) 0.9\% picric acid, 24\% (v/v) $37 \%$ formaldehyde, and 5\% (v/v) acetic acid). Diluted Bouin's fixed testes, total testis RNA, and RNA isolated from A spermatogonia (93\% pure) of VAD rats were obtained during previous studies (van Pelt etal. 1996, 2002). The A spermatogonia were, as described previously, isolated by enzymatic digestion and percoll gradient centrifugation. The purity was determined by Nomarski interference microscopy (van Pelt et al. 1996). All the procedures were performed according to the regulations provided by the ethical committee of the University of Utrecht that also approved of the experiments.

\section{DNA sequencing and RT-PCR}

In order to determine the rat Utf1 sequence, primers were designed by comparing the sequence of human and mouse (accession numbers AB011076 and NM_009482 respectively) mRNA. RT-PCR was performed on cDNA from total testis of an adult rat. DNA fragments of $\sim 1000 \mathrm{bp}$ were isolated from gel and subcloned into pcDNA3.1/N5-His TOPO vector (Invitrogen). Plasmid DNA of two clones was prepared for DNA sequence 
analysis. DNA sequence analyses were performed on automated ABI PRISM 310 and 377 DNA sequencers, using Dye Terminator cycle sequencing chemistry (all from Applied Biosystems, Foster city, CA, USA). Sequence alignment was performed using ClustalW and Genedetect (Chenna et al. 2003; http://www.ebi. ac.uk/embnet.news/vol4_2/genedoc.html). Amino acid identity and similarity to mouse and human UTF1 sequences were determined with MatGAT 2.0 (Campanella et al. 2003). RT-PCR on cDNA of total testes of rats of different ages was performed with the following primers: Utf1 fw: $5^{\prime}$-cgctcagattcagctcttcc-3', rv: 5'-cgaaggaacctcgtagatgc-3' (333 bp); Pou5f1 fw: 5'-cgaggag tcccaggatatga-3', rv: 5' -gttccacctcacacggttct-3' (334 bp); Sox2 fw: $5^{\prime}$-accagctcgcagacctacat- $3^{\prime}$, rv: $5^{\prime}$-ccctcccaattcccttgtat- $3^{\prime}$ (388 bp); and Zbtb16 fw: 5'-tctgtctgctgtgtgggaag-3', rv: 5'-gtggcagagttgcactcaa-3' (353 bp).

\section{Immunohistochemistry}

For immunolocalization of UTF1 and ZBTB16, adjacent $5 \mu \mathrm{m}$ sections of diluted Bouin's fluid fixed testes were mounted on TESPA-coated glass slides and dried overnight at $37^{\circ} \mathrm{C}$. For antigen retrieval, sections were boiled in a microwave oven (H2500, Bio-Rad) at $98^{\circ} \mathrm{C}$ in $0.01 \mathrm{M}$ sodium citrate ( $\mathrm{pH}$ 6.0). Sections for localization of ZBTB16 were boiled three times for $10 \mathrm{~min}$. For localization of UTF1, the boiling time depended on the antibody used one time for $10 \mathrm{~min}$ (AB3383, Chemicon International, Temecula, CA, USA; ab24273, Abcam, Cambridge, UK and an antibody generated by Okuda et al. (1998) or 20 min (antibody kindly provided by Dr B J Eggen; Chuva de Sousa Lopes et al. 2005, van den Boom et al. 2007). Endogenous peroxidase was blocked with $0.35 \% \mathrm{H}_{2} \mathrm{O}_{2}$ in PBS for $15 \mathrm{~min}$. After blocking in 5\% normal goat serum or normal horse serum (NHS; Vector Laboratories, Burlingame, CA, USA), slides were incubated overnight at $4{ }^{\circ} \mathrm{C}$ with $1: 100$ UTF1 (AB3383, ab24273, antibody from Dr Okuda), 1:200 (UTF1 antibody from Dr Eggen), or 1:50 ZBTB16 (OP128, Calbiochem, San Diego, CA, USA) antibody in $0.1 \%$ BSAc in PBS (Aurion, Wageningen, The Netherlands). Secondary biotinylated goat anti-rabbit (BA-1000, Vector Laboratories) or horse anti-mouse (PK6102, Vector Laboratories) was used 1:200 in $0.1 \%$ BSAc in PBS for $1 \mathrm{~h}$ at RT. Horseradish peroxidase avidinbiotin complex reaction was performed according to the manufacturer's protocol (PK6102, Vector Laboratories). Bound antibody was finally detected by diaminobenzidine (Sigma). Sections were counterstained with Mayer's hematoxylin, dehydrated, and mounted with Pertex (Cellpath Ltd, Hemel Hempstead, UK). For negative control, the first antibody was replaced with normal rabbit (sc-2025, Santa Cruz Biotechnology Inc., Santa Cruz, CA, USA) or normal mouse IgG (sc-2027, Santa Cruz Biotechnology Inc.) for UTF1 and ZBTB16 respectively. Figure 3 shows UTF1 immunolocalization with AB3383 antibody.

\section{Cell counts and staging}

Cells expressing UTF1 and/or ZBTB16 were counted in the testes of rats at various ages. Cell numbers were expressed per tubule cross-section. Cell counts were performed until at least 100 tubule cross-sections per testis were scored. Numbers were expressed as means \pm S.E.M. Statistical analysis (paired $t$-test) was performed with Graphpad Prism 4 (Graphpad Software, San Diego, CA, USA). Adjacent sections were stained with periodic acid Schiff's reagent and Mayer's hematoxylin in order to determine the epithelial stage of the tubule cross-sections.

\section{Immunofluorescence}

Co-immunolocalization of UTF1 and ZBTB16 was studied in diluted Bouin's fluid fixed testes of rats at 42, 56, and $70 \mathrm{dpp}$. Five micrometer paraffin sections were mounted on TESPAcoated glass slides and dried overnight at $37^{\circ} \mathrm{C}$. For antigen retrieval, the sections were boiled at $98{ }^{\circ} \mathrm{C}$ in $0.01 \mathrm{M}$ sodium citrate for $10 \mathrm{~min}$. The sections were blocked with $5 \%$ NHS in $0.1 \%$ BSAc and incubated overnight at $4{ }^{\circ} \mathrm{C}$ with $1: 100$ UTF1 (AB3383, Chemicon International) and 1:50 ZBTB16 (OP128, Calbiochem) antibodies in $0.1 \%$ BSAc. To fluorescently label the UTF1-positive cells, slides were incubated with 1:100 goat anti-rabbit Alexa 488 (A11008, Molecular Probes, Leiden, The Netherlands) in $0.1 \%$ BSAc for $1 \mathrm{~h}$. ZBTB16 antibody was labeled by incubation with 1:200 biotinylated horse antimouse (PK6102, Vector Laboratories) for $1 \mathrm{~h}$, followed by incubation with 1:200 streptavidin Alexa Fluor 495 conjugated (S11227, Molecular Probes) in $0.1 \%$ BSAc. The sections were mounted with Vectashield (Vector Laboratories) and viewed with a Leitz DMIRB fluorescence microscope (Leica, Voorburg, The Netherlands).

\section{Acknowledgements}

This study was supported by the European Science Foundation Programme EuroSTELLS, trough ZonMW 910-20-032. The authors declare that there is no conflict of interest that would prejudice the impartiality of this scientific work.

\section{References}

Aponte PM, van Bragt MP, de Rooij DG \& van Pelt AM 2005 Spermatogonial stem cells: characteristics and experimental possibilities. Acta Pathologica, Microbiologica, et Immunologica Scandinavica 113 727-742.

van den Boom V, Kooistra SM, Boesjes M, Geverts B, Houtsmuller AB, Monzen K, Komuro I, Essers J, Drenth-Diephuis LJ \& Eggen BJL 2007 UTF1 is a chromatin-associated protein involved in ES cell differentiation. Journal of Cell Biology 178 913-924.

Buaas FW, Kirsh AL, Sharma M, McLean DJ, Morris JL, Griswold MD, de Rooij DG \& Braun RE 2004 Plzf is required in adult male germ cells for stem cell self-renewal. Nature Genetics 36 647-652.

Campanella JJ, Bitincka L \& Smalley J 2003 MatGAT: an application that generates similarity/identity matrices using protein or DNA sequences. BMC Bioinformatics 429.

Chenna R, Sugawara H, Koike T, Lopez R, Gibson TJ, Higgins DG \& Thompson JD 2003 Multiple sequence alignment with the Clustal series of programs. Nucleic Acids Research 31 3497-3500.

Chuva de Sousa Lopes SM, van den Driesche S, Carvalho RL, Larsson J, Eggen B, Surani MA \& Mummery CL 2005 Altered primordial germ cell migration in the absence of transforming growth factor beta signaling via ALK5. Developmental Biology 284 194-203.

Costoya JA, Hobbs RM, Barna M, Cattoretti G, Manova K, Sukhwani M, Orwig KE, Wolgemuth DJ \& Pandolfi PP 2004 Essential role of Plzf in maintenance of spermatogonial stem cells. Nature Genetics 36 653-659. 
Fukushima A, Okuda A, Nishimoto M, Seki N, Hori TA \& Muramatsu M 1998 Characterization of functional domains of an embryonic stem cell coactivator UTF1 which are conserved and essential for potentiation of ATF-2 activity. Journal of Biological Chemistry 273 25840-25849.

Guan K, Nayernia K, Maier LS, Wagner S, Dressel R, Lee JH, Nolte J, Wolf F, Li M, Engel W \& Hasenfuss G 2006 Pluripotency of spermatogonial stem cells from adult mouse testis. Nature 440 1199-1203.

Hofmann MC, Braydich-Stolle L \& Dym M 2005 Isolation of male germ-line stem cells; influence of GDNF. Developmental Biology 279 114-124.

Huckins C 1971 The spermatogonial stem cell population in adult rats. I. Their morphology, proliferation and maturation. Anatomical Record 169 533-557.

Kanatsu-Shinohara M, Inoue K, Lee J, Yoshimoto M, Ogonuki N, Miki H, Baba S, Kato T, Kazuki Y, Toyokuni S et al. 2004 Generation of pluripotent stem cells from neonatal mouse testis. Cell 119 1001-1012.

Matsui Y, Zsebo K \& Hogan BL 1992 Derivation of pluripotential embryonic stem cells from murine primordial germ cells in culture. Cell $\mathbf{7 0}$ 841-847.

Mitranond V, Sobhon P, Tosukhowong P \& Chindaduangrat W 1979 Cytological changes in the testes of vitamin-A-deficient rats. I. Quantitation of germinal cells in the seminiferous tubules. Acta Anatomica 103 159-168.

Nishimoto M, Fukushima A, Okuda A, Muramatsu M, Seki N, Hori TA, Orimo A, Yamagishi T, Nabeshima Y, Kuro-o M et al. 1999 The gene for the embryonic stem cell coactivator UTF1 carries a regulatory element which selectively interacts with a complex composed of Oct-3/4 and Sox-2. Molecular and Cellular Biology 19 5453-5465.

Nishimoto M, Miyagi S, Yamagishi T, Sakaguchi T, Niwa H, Muramatsu M \& Okuda A 2005 Oct-3/4 maintains the proliferative embryonic stem cell state via specific binding to a variant octamer sequence in the regulatory region of the UTF1 locus. Molecular and Cellular Biology 25 5084-5094.

Okuda A, Fukushima A, Nishimoto M, Orimo A, Yamagishi T, Nabeshima $Y$, Kuro-o M, Boon K, Keaveney M, Stunnenberg HG et al. 1998 UTF1, a novel transcriptional coactivator expressed in pluripotent embryonic stem cells and extra-embryonic cells. EMBO Journal 17 2019-2032. van Pelt AM \& De Rooij DG 1990 The origin of the synchronization of the seminiferous epithelium in vitamin A-deficient rats after vitamin A replacement. Biology of Reproduction 42 677-682.

van Pelt AM, Morena AR, van Dissel-Emiliani FM, Boitani C, Gaemers IC, de Rooij DG \& Stefanini M 1996 Isolation of the synchronized A spermatogonia from adult vitamin A-deficient rat testes. Biology of Reproduction 55 439-444.

van Pelt AM, Roepers-Gajadien HL, Gademan IS, Creemers LB, de Rooij DG \& van Dissel-Emiliani FM 2002 Establishment of cell lines with rat spermatogonial stem cell characteristics. Endocrinology 143 1845-1850.

Pesce M, Wang X, Wolgemuth DJ \& Scholer H 1998 Differential expression of the Oct-4 transcription factor during mouse germ cell differentiation. Mechanisms of Development 71 89-98.

de Rooij DG 1998 Stem cells in the testis. International Journal of Experimental Pathology 79 67-80.

de Rooij DG \& Russell LD 2000 All you wanted to know about spermatogonia but were afraid to ask. Journal of Andrology 21 776-798.

Seandel M, James D, Shmelkov SV, Falciatori I, Kim J, Chavala S, Scherr DS, Zhang F, Torres R, Gale NW et al. 2007 Generation of functional multipotent adult stem cells from GPR125 + germline progenitors. Nature 449 346-350.

Shamblott MJ, Axelman J, Wang S, Bugg EM, Littlefield JW, Donovan PJ, Blumenthal PD, Huggins GR \& Gearhart JD 1998 Derivation of pluripotent stem cells from cultured human primordial germ cells. PNAS 95 13726-13731.

Shi YQ, Wang QZ, Liao SY, Zhang Y, Liu YX \& Han CS 2006 In vitro propagation of spermatogonial stem cells from KM mice. Frontiers in Bioscience 11 2614-2622.

Smith A 2006 A glossary for stem-cell biology. Nature 4411060.

Received 28 November 2007

First decision 21 January 2008

Revised manuscript received 17 March 2008

Accepted 27 March 2008 